\title{
Gasdermin D mediates inflammation-induced defects in reverse cholesterol transport and promotes atherosclerosis.
}

Emmanuel Opoku1 $\uparrow$, C. Alicia Traughber, ${ }^{1,2,3} \uparrow$, David Zhang ${ }^{1}$, Amanda J lacano ${ }^{1}$, Mariam Khan ${ }^{2,3}$, Juying $\operatorname{Han}^{1}$, Jonathan D Smith ${ }^{1,}$ and Kailash Gulshan*1,2,3

${ }^{1}$ Department of Cardiovascular and Metabolic Sciences, Cleveland Clinic, Cleveland OH 44195, USA.

${ }^{2}$ Center for Gene Regulation in Health and Disease, Department of Biological, Geological, and

Environmental Sciences, College of Science and Health Professions, Cleveland State University,

Cleveland, $\mathrm{OH} 44115$, USA.

${ }^{3}$ Department of Biological, Geological and Environmental Sciences, Cleveland State University,

Cleveland $\mathrm{OH} 44115$, USA.

†Contributed equally.

${ }^{*}$ Correspondence to: Kailash Gulshan, Ph.D.

Email: k.gulshan@csuohio.edu

Key Words: Cholesterol, Cardiovascular disease, Atherosclerosis, Inflammation, NIrp3 inflammasome, Gasdermin D, IL-1 1 , $\mathrm{PIP}_{2}$.

Total Word count: 8,219

Word count of abstract: 257

Total characters with spaces: 54,214

Numbers of figures: 7

TOC category: Basic, TOC subcategory: Atherosclerosis 


\section{Abstract:}

Activation of inflammasomes, such as NIrp3 and Aim2, can exacerbate atherosclerosis in mice and humans. Gasdermin D (GsdmD) serves as a final executor of inflammasome activity, by generating membrane pores for the release of mature Interleukin-1beta (IL-1ß). Inflammation dampens reverse cholesterol transport (RCT) and promotes atherogenesis, while anti-IL-1 $\beta$ antibodies were shown to reduce cardiovascular disease in humans. Though NIrp3/AIM2 and $\mathrm{IL}-1 \beta$ nexus is an emerging atherogenic pathway, the direct role of GsdmD in atherosclerosis is not yet fully clear. Here, we used in-vivo NIrp3 inflammasome activation to show that the GsdmD1- mice release $\sim 80 \%$ less IL-1 $\beta$ vs WT mice. The GsdmD ${ }^{-1-}$ macrophages were more resistant to NIrp3 inflammasome mediated reduction in cholesterol efflux, showing $\sim 26 \%$ decrease vs. $\sim 60 \%$ reduction in WT macrophages. GsdmD expression in macrophages exacerbated foam cell formation in an IL-1 $\beta$ dependent fashion. The $\mathrm{GsdmD}^{-/}$mice were resistant to Nlrp3 inflammasome mediated defect in RCT, with $\sim 32 \%$ reduction in plasma RCT vs. $\sim 57 \%$ reduction in WT mice, $\sim 17 \%$ reduction in RCT to liver vs. $42 \%$ in WT mice, and $\sim 37 \%$ decrease in RCT to feces vs. $\sim 61 \%$ in WT mice. The LDLr anti-sense oligonucleotides (ASO) induced hyperlipidemic mouse model showed the role of GsdmD in promoting atherosclerosis. The GsdmD ${ }^{-/-}$mice exhibit $\sim 42 \%$ decreased atherosclerotic lesion area in females and $\sim 33 \%$ decreased lesion area in males vs. WT mice. The atherosclerotic plaque-bearing sections stained positive for the cleaved N-terminal fragment of GsdmD, indicating cleavage of GsdmD in atherosclerotic plaques. Our data show that GsdmD mediates inflammation-induced defects in RCT and promotes atherosclerosis.

\section{Summary: GsdmD mediates inflammation-induced defects in RCT and promotes atherosclerosis.}




\section{Non-standard abbreviations and acronyms:}

AcLDL, acetylated-low-density lipoprotein

AIM2, absent in melanoma 2

ASO, antisense oligonucleotides

CAD, coronary artery disease

CANTOS, canakinumab anti-inflammatory thrombosis outcomes study

CVD, cardiovascular disease

GsdmD, gasdermin D

IL-1 $\beta$, interleukin 1-beta

IL-18, interleukin 18

LDLr, low-density lipoprotein receptor

MACE, major adverse coronary events

NLRP3, nod-like receptor family pyrin domain-containing 3

NETs, neutrophil extracellular traps

(oxLDL. oxidized low-density lipoprotein

PIP2, phosphatidylinositol 4,5-bisphosphate

PS, phosphatidylserine

TLR, toll-like receptor 


\section{Introduction:}

The NLRP3 inflammasome is implicated in promoting cardiovascular disease (CVD) and metabolic diseases such as obesity-induced inflammation/insulin resistance $(1,2)$, and destabilization of atherosclerotic plaques $(1,3)$. The Canakinumab Anti-Inflammatory Thrombosis Outcomes Study (CANTOS) trial showed that anti-IL-1 $\beta$ therapy reduced major adverse coronary events (MACE), independent of lipid levels (4). The NIrp3 inflammasome target protein "GsdmD" is involved in multiple pro-inflammatory pathways such as the release of IL-1 $\beta$, pyroptotic cell death, and formation of neutrophil extracellular traps/NETosis $(5,6)$. NIrp3 inflammasome is activated in advanced human atherosclerotic plaques, but the role of GsdmD in sterile inflammatory diseases such as atherosclerosis is not yet described. Interestingly, GsdmD cleavage does not necessarily lead to pyroptotic cell death under all conditions, as GsdmD also plays a role in the release of IL-1 $\beta$ from living macrophages (7-9). Thus living, but inflamed, macrophages in atherosclerotic plaques may contribute to elevating IL-1 $\beta$ levels in GsdmD dependent manner.

Deposition and oxidative modification of low-density lipoprotein-cholesterol (LDL-C) in the arterial intima promotes monocyte entry and transformation into macrophages, leading to lipid engulfment and foam cell formation. Foam cells become dysfunctional over time due to unregulated lipid uptake, leading to fatty streaks and further amplification of inflammation and progression of atherosclerosis $(1,10-12)$. Removal of excess cholesterol from artery wall macrophages via reverse cholesterol transport (RCT) may reverse the progression of atherosclerosis $(13,14)$. Chronic inflammation serves as a double-edged sword by promoting the continued influx of immune cells into the plaque area via inducing the expression of adhesion molecules such as vascular cell adhesion molecule-1 (VCAM-1) on endothelial cells, and by dampening the protective RCT pathway $(15,16)$. Accumulation of oxidized lipids and 
cholesterol crystals in plaques can engage the toll-like receptor (TLR) pathway and induce the assembly of the NLRP3 inflammasome $(1,3,12,17)$. The NLRP3 inflammasome plays a key role in processing procaspase 1 to active caspase 1 , which in turn can mediate the cleavage of pro-interleukin-1 $\beta$ (pro-IL-1 $\beta$ ), pro-interleukin-18 (pro-IL-18), and Gasdermin D (GsdmD) to generate active IL-1 $\beta$, active IL-18, and active N-terminal fragment of GsdmD (GsdmD-NT). Mature IL-1 $\beta$ can be released from inflamed macrophages in at least two ways. In one pathway, the cleaved GsdmD-NT binds to phosphatidylinositol lipids (PIPs) and phosphatidylserine (PS) on the inner leaflet of the plasma membrane of cells to generate pores for the fast release of mature IL-1 $\beta(18-20)$. In the second pathway, the cleaved IL-1 $\beta$ exit via directly binding to the phosphatidylinositol 4,5-bisphosphate (PIP2) in plasma membrane lipid rafts (21). In contrast to NIrp3 inflammasome and IL-1 $\beta$, the direct role of GsdmD in atherosclerosis is not yet clear. Though GsdmD cleavage and ensuing membrane blebbing and pyroptosis are generally associated with the robust release of cytokines and clearance of microbial infection, it's important to note that GsdmD also plays a role in IL-1 $\beta$ release from living macrophages (22). Furthermore, similar to the apoptotic death of cholesterol-loaded macrophages $(23,24)$, the pyroptotic cell death can be induced by cholesterol crystals mediated NIrp3 inflammasome activation in advanced human atherosclerotic plaques $(1,25)$. Thus, GsdmD may play role in NIrp3 inflammasome mediated dampening of RCT and in promoting the progression of atherosclerosis, either by virtue of increasing IL-1 $\beta$ release from live inflamed immune cells or by promoting pyroptotic cell death in advanced atherosclerotic plaques. 


\section{Results:}

\section{GsdmD $^{-/-}$mice release lower levels of IL-1 $\beta$ upon in-vivo NIrp3 inflammasome assembly.}

To confirm that $\mathrm{GsdmD}^{-/}$macrophages are defective in IL-1 $\beta$ release, primary bone-marrowderived macrophages (BMDMs) isolated from wild type (WT) or GsdmD ${ }^{-/-}$mice were primed with $1 \mu \mathrm{g} / \mathrm{ml}$ LPS for $4 \mathrm{~h}$. To induce NIrp3 inflammasome assembly, these cells were incubated with $5 \mathrm{mM}$ ATP for 20 min or $1 \mu \mathrm{M}$ Nigericin for $1 \mathrm{~h}$, followed by a collection of cell-free media to measure released IL-1 $\beta$. Consistent with previous studies, the GsdmD ${ }^{-/-}$macrophages showed a $\sim 80 \%$ decrease in the release of IL-1 $\beta$ as compared to control macrophages (Fig. 1A). The basal as well as the LPS induced expression levels of NLRP3 and pro-IL-1 $\beta$ were not altered in $\mathrm{GsdmD}^{-/-}$BMDMs, indicating that only IL-1 $\beta$ release is defective (Fig $1 B$ ).

To determine the role of GsdmD in NIrp3 inflammasome mediated IL-1 $\beta$ release in-vivo, the Nlrp3 inflammasome was induced in mice by i.p. injection of $5 \mu \mathrm{g}$ LPS, followed $4 \mathrm{~h}$ later with i.p injection of $0.5 \mathrm{ml}$ of $30 \mathrm{mM}$ ATP. Control mice received either saline or LPS + saline injections. The peritoneal lavage fluid was collected $30 \mathrm{~min}$ after ATP injection and analyzed for IL-1 $\beta$ levels by ELISA. As shown in Fig. 1C, WT mice injected with LPS and ATP showed robust IL-1 $1 \beta$ levels in peritoneal lavage while the $\mathrm{GsdmD}^{-/-}$mice had a $\sim 90 \%$ reduction in IL$1 \beta$ levels. The IL-1 $\beta$ levels were not detectable in peritoneal lavage of $\mathrm{WT}$ or $\mathrm{GsdmD}^{-/-}$mice injected with saline or LPS alone (Fig. 1D), showing that both LPS and ATP are required for in vivo NIrp3 inflammasome activation and GsdmD is required for IL-1 $\beta$ release.

\section{GsdmD ${ }^{-/-}$macrophages showed protection from NIrp3 inflammasome-induced defect in} cholesterol efflux. To determine if NIrp3 inflammasome activation leads to defective cholesterol efflux and if GsdmD deletion protects against this effect, cholesterol efflux assays were performed in BMDMs \pm induction of Nlrp3 inflammasome. The BMDMs were labeled with 
${ }^{3} \mathrm{H}$ cholesterol for $24 \mathrm{~h}$, followed by induction of ABCA1 expression with liver $\mathrm{X}$ receptor (LXR) agonist T-0901317. The macrophages were treated \pm LPS and Nigericin, followed by a chase with $5 \mu \mathrm{g} / \mathrm{ml}$ lipid-free apoA1 for $4 \mathrm{~h}$. As shown in Fig. 2A, LPS/Nigericin treatment of WT BMDMS led to $\sim 60 \%$ decrease in cholesterol efflux vs. untreated BMDMs. The BMDMs derived from GsdmD ${ }^{-/-} \mathrm{KO}$ mice showed much lower inhibition of cholesterol efflux ( 26\% decrease vs. untreated). The direct effect of IL-1 $\beta$ was evaluated by treatment of LPS treated WT or GsdmD ${ }^{-/-}$BMDMs with exogenous recombinant mouse IL-1 $\beta$. The addition of exogenous recombinant mouse IL-1 $\beta$ inhibited cholesterol efflux in both WT and GsdmD ${ }^{-/-}$ BMDM (Fig. S1). These data indicated that exogenous IL-1 $\beta$ is sufficient to drive GsdmD ${ }^{-/-} \mathrm{KO}$ macrophages to respond similarly to WT macrophages. Thus, IL-1 $\beta$ appears to be a major factor responsible for inhibiting cellular cholesterol efflux. To recapitulate the environment of atherosclerotic plaque area, where IL-1 $\beta$ released from inflamed foam cells may affect the cholesterol efflux capacity of neighboring cells, we used the conditioned media from BMDMs treated with LPS/ATP. The conditioned media from WT BMDMs inhibited cholesterol efflux in both WT and GsdmD ${ }^{-/-} \mathrm{KO}$ macrophages, while the conditioned media from GsdmD ${ }^{-/-}$BMDMs treated with LPS/ATP showed reduced suppression of cholesterol efflux (Fig. 2B, S1). These data indicated that macrophages lacking GsdmD are more resistant to inflammasome-induced reduction in cholesterol efflux, mostly due to defective IL-1 $\beta$ release. The ABCA1 expression was pre-induced with T-compound, and as expected no significant differences in ABCA1 levels were observed in control or conditioned-media treated macrophages (Fig. S2). Next, we tested if IL-1 $\beta$ is interfering with apoA1 binding to the cell membrane. The apoA1 binding to the cellsurface \pm WT conditioned media was probed by using an Alexa647 labeled apoA1 as described before $(26,27)$. The BMDMs showed basal apoA1 binding as they express ABCA1, albeit at a low level, while induction of ABCA1 with T compound led to a significant increase in 
apoA1 binding to the cell surface (Fig. 2C. 2D). Treatment of BMDMs with WT-CM led to significant decrease in apoA1 binding to the cell surface (Fig. 2C, 2D), while the $\mathrm{GsdmD}^{-/-} \mathrm{CM}$ media showed no effect on apoA1 binding to the cell surface (Fig. 2D).

PIP2 localization modulated GsdmD-NT mediated cell lysis. PIP2 serves as a ligand for GsdmD N-terminal (GsdmD-NT) fragment and transfection of GsdmD-NT in HEK293 cells promotes cell lysis (18). ABCA1 expression reduces PIP2 on the inner leaflet of plasma membrane $(27,28)$, thus we tested if ABCA1 expression can reduce GsdmD mediated cell lysis by transfecting the control HEK293 and HEK293-ABCA1 cells with GsdmD-NT. Transfection of GsdmD-NT, but not the full-length GsdmD, caused cell lysis in HEK293 cells (Fig. 2E). The HEK293 cells expressing ABCA1 showed reduced cell lysis (Fig. 2E), indicating that PIP2 flop across the cell membrane can dampen GsdmD-NT mediated pore-formation and cell lysis. To test the direct role of PIP2 in GsdmD-NT cell lysis, we generated HEK293 cells stably expressing GFP tagged Pleckstrin homology domain of Phospholipase-C protein (2XPH-PLC-eGFP). As the majority of cellular PIP2 is localized to the inner leaflet of plasma membrane, as expected the PH-PLC-eGFP signal was localized at the plasma membrane (Fig. S3). The HEK293-2X-PH-PLC-eGFP cells transfected with GsdmD-NT showed reduced lysis vs. control HEK293 cells (Fig. 2E). The membrane recruitment of N-terminal GsdmD was confirmed by fractionation of cytosolic and membrane proteins using the sequential fractionation method as described earlier (29). The membrane recruitment of GsdmD-NT was reduced in HEK-ABCA1 cells and HEK-PH-PLC cells vs. control HEK293 cells (Fig. 2F, 2G). The control HEK293 cells showed $\sim 80 \%$ of GsdmD-NT recruitment to the membrane while the rest $\sim 18 \%$ remained in cytoplasm. In contrast, the HEK-ABCA1 and HEK-PH-PLC cells showed $\sim 40 \%$ and $\sim 35 \%$ recruitment of GsdmD-NT to the membrane, while $\sim 60 \%$ and 
$65 \%$ remains in cytoplasm. These data indicated that blocking PIP2 access to NT-GsdmD reduced recruitment of GsdmD-NT to the plasma membrane, leading to lower cell lysis.

GsdmD promotes foam cell formation. To determine if the IL-1 $\beta$ released from inflamed cells can promote foam cell formation, the lipid loading was performed along with treatment with conditioned media. The WT BMDMs were incubated with the conditioned media isolated from LPS+ATP treated WT or GsdmD $/$ - macrophages along with $25 \mu \mathrm{g} / \mathrm{ml}$ Acetylated-lowdensity lipoprotein (AcLDL) and $25 \mu \mathrm{g} / \mathrm{ml}$ oxidized low-density lipoprotein (oxLDL) for $48 \mathrm{~h}$ to induce foam cell formation. The BMDMs were washed thoroughly with serum-free media and stained with lipid-binding Nile-red dye, followed by either qualitative analysis via fluorescent microscopy or quantitative analysis via flow cytometry. The WT BMDMs, as well as the GsdmD ${ }^{-/-}$BMDMs, showed higher lipid accumulation in presence of WT conditioned media (Fig. 3A, Fig. S5 ). In contrast, the WT or GsdmD ${ }^{-/}$BMDMs showed reduced foam cell formation with GsdmD ${ }^{-/-}$conditioned media treatment (Fig. 3A, 3B). These data indicated that GsdmD mediated IL-1 $\beta$ release promotes foam cell formation. Since the GsdmD ${ }^{-1-}$ macrophages are resistant to pyroptotic cell death, we sought to determine if lipid-laden GsdmD ${ }^{-/}$can instead undergo apoptotic cell death. Foam cell formation was induced by loading WT or GsdmD ${ }^{-1-}$ BMDMs with $100 \mu \mathrm{g} / \mathrm{ml}$ AcLDL for 48h, followed by Nlrp3 inflammasome activation by LPS and ATP treatment. The cells were washed with PBS and the phosphatidylserine (PS) exposure at the cell surface was determined by flow cytometry based Annexin-Cy5 binding assay as described earlier(27). As shown in Fig. 3C, the GsdmD ${ }^{-1-}$ foam cells showed a much higher annexin $V$ signal vs. WT foam cells $(N=4$, mean $\pm S D)$. These data indicated that the lack of GsdmD in Nlrp3 inflammasome-induced macrophages can promote apoptotic cell death in lieu of pyroptosis. 


\section{GsdmD ${ }^{-/-}$mice showed protection from NIrp3 inflammasome-induced defect in RCT. To}

determine if NIrp3 inflammasome assembly in mice leads to defective RCT and if GsdmD ${ }^{-/-} \mathrm{KO}$ mice are protected against this effect, an RCT assay was performed as described earlier (16). The BMDMs from WT mice were loaded with acetylated-low-density lipoprotein (Ac-LDL) and radiolabeled ${ }^{3} \mathrm{H}$ cholesterol for $48 \mathrm{~h}$ to generate foam cells. The WT and GsdmD KO mice pretreated with LPS + ATP treatment were injected with foam cells, following the RCT assay design shown in Fig. $\mathbf{4 A}$. The radioactive cholesterol efflux to plasma was determined by collection of blood samples at $24 \mathrm{~h}, 48 \mathrm{~h}$, and $72 \mathrm{~h}$. The liver and feces samples were collected post euthanasia and processed to determine the \% of cholesterol efflux. As shown in Fig. 4B, NIrp3 inflammasome activation reduces RCT to plasma in WT mice by $67 \%$ at $24 \mathrm{~h}$, by $33 \%$ at $48 \mathrm{~h}$, by $47 \%$ at $72 \mathrm{~h}$ (Fig. $2 \mathrm{~B}$ ), while $\mathrm{GsdmD}^{-/-}$mice showed only $46 \%$ reduction at $24 \mathrm{~h}, 26 \%$ at $48 \mathrm{~h}$, and $33 \%$ reduction at $72 \mathrm{~h}$ (Fig. $\mathbf{4 C}$ ). The cumulative reduction in RCT to plasma in WT mice was $\sim 57 \%$ vs. $32 \%$ in $\mathrm{GsdmD}^{-/-}$mice, showing that the $\mathrm{GsdmD}^{-/-}$mice have $25 \%$ more preserved RCT to plasma vs. WT mice (Fig. 4D). The RCT to liver was reduced by $42 \%$ in WT mice vs. $17 \%$ reduction in GsdmD ${ }^{-/-}$mice (Fig. $4 E$ ). The RCT to feces was reduced upon NIrp3 inflammasome activation by $31 \%$ at $24 \mathrm{~h}$, by $77 \%$ at $48 \mathrm{~h}$, by $63 \%$ at $72 \mathrm{~h}$ in WT mice

(Fig. 4F), while GsdmD ${ }^{-/-}$mice showed $18 \%$ reduction at $24 \mathrm{~h}, 40 \%$ at $48 \mathrm{~h}$, and $38 \%$ reduction at $72 \mathrm{~h}$ (Fig. 4G). The cumulative reduction in RCT to feces in WT mice was $\sim 61 \%$ vs. $37 \%$ in GsdmD ${ }^{-/-}$mice, showing that GsdmD ${ }^{-/-}$mice have $24 \%$ more preserved RCT to feces vs. WT mice upon NIrp3 inflammasome induction (Fig. 4H). These data indicated that GsdmD promotes inflammation-induced defects in reverse cholesterol transport.

LDLr ASO induces hyperlipidemia in WT and GsdmD-/- mice. Antisense oligonucleotides directed to LDLR mRNA cause hypercholesterolemia in western type diet (WTD) fed wild-type C57BL/6 mice (30). The 12-week-old WT and GsdmD ${ }^{-/-}$KO mice were injected i.p with LDLr 
ASO or control ASO once a week for 9 weeks and mice were fed an atherogenic WTD throughout the study, as shown in the atherosclerosis study design (Fig. 5A). The mice were weighed and plasma was collected before and after the 9-week ASO treatments, and total cholesterol (TC) levels were determined. As shown in Fig. 5B, no significant differences were found in body weight or cholesterol levels in WT and GsdmD ASO and diet interventions. The plasma TC levels showed a robust increase in LDLr ASO treated female mice and reached an average level of $\sim 800 \mathrm{mg} / \mathrm{dl}$ (Fig. 5C). The male mice also showed similar trends with no difference in body weight gain and increase in plasma cholesterol upon LDLr ASO treatment (Fig. 5D, 5E). These data showed that the LDLr ASO has equivalent efficacy in both WT and GsdmD-/- mice.

GsdmD ${ }^{-/-}$mice showed reduced atherosclerosis. LDLr ASO treatment and WTD feeding lead to the development of atherosclerotic lesions in the aortic root, aortic arch, and brachiocephalic artery (30). To determine if GsdmD plays a role in the progression of atherosclerosis, the 12-week-old WT and GsdmD"-- mice were injected i.p with LDLr ASO or control ASO once a week, and fed with atherogenic western-type diet (WTD), to generate hyperlipidemia. The mice were sacrificed at the end of week 21 and hearts were perfused with PBS, followed by harvesting of heart, spleen, and liver. The knockdown of hepatic LDLr by the LDLr ASO was confirmed by the western blot of liver extracts (Fig. 6A).

The hearts were sectioned and the atherosclerotic plaques were quantified using the oil red $\mathrm{O}$ positive aortic root atherosclerotic lesion area. As shown in Fig. 6B, WT mice injected with LDLr ASO showed large atherosclerotic lesions in both males and females, while the GsdmD ${ }^{-/-}$ mice showed significantly smaller atherosclerotic lesions with $\sim 42 \%$ decreased lesion area in females (Fig. 6C) and $~ 33 \%$ decreased lesion area in males (Fig. 6D), The WT and GsdmD mice injected with control ASO and fed with WTD showed no atherosclerotic plaques (Fig. S5). 


\section{GsdmD is cleaved in atherosclerotic plaques and play role in inducing expression of}

adhesion molecule. To determine if GsdmD is actively cleaved in plaques, we prepared protein extracts from atherosclerotic aortas and performed western blot analysis using the antibody that recognizes only cleaved GsdmD and not the full-length GsdmD. As shown in

Fig. 7A, the cleaved GsdmD fragment was detected in the atherosclerotic aortas of WT mice. As expected, the protein extracts of aortas from GsdmD ${ }^{-/-}$mice showed no positive signal. To investigate the mechanism of GsdmD in promoting atherosclerosis, we also probed the aortic protein extracts for VCAM-1 expression levels. The LDLr ASO injected mice showed higher expression of VCAM-1 in aortic extracts vs. control ASO injected mice (Fig. 7A). The aortic extracts from LDLr ASO injected GsdmD/- mice showed lower induction in levels of VCAM-1 vs. WT mice, indicating that GsdmD expression induces VCAM-1 expression in hyperlipidemic mice. To further confirm the presence of cleaved GsdmD in atherosclerotic plaque areas, we performed immunohistochemistry on aortic sections and stained the tissue sections with cleaved GsdmD antibody and counterstained with hematoxylin. As shown in Fig. 7B, the aortic sections from the LDLr ASO injected WT mice showed a positive signal with cleaved GsdmD antibody while sections from LDLr ASO injected GsdmD ${ }^{-/-}$mice showed no staining. The plasma ATP levels were shown to be significantly higher in atherosclerotic LDLR $^{-/-}$mice vs.

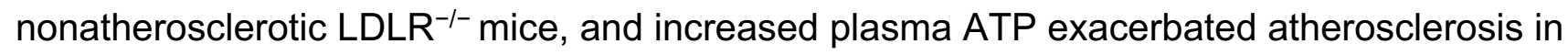
LDLr $^{-/-}$mice (31). The increased plasma ATP levels can serve as a danger signal and induce NIrp3 inflammasome assembly and IL-1 $\beta$ release in plaques. Increased IL-1 $\beta$ levels can promote increased recruitment of immune cells to plaque area and promote increased ATP release from damaged foam cells due to the release of cytoplasmic content. To determine if the GsdmD pathway contribute to ATP release in atherosclerotic LDLR ${ }^{-/-}$mice, the plasma ATP levels were determined in WT and GsdmD ${ }^{-/-}$mice injected with LDLr ASO and fed with 
atherogenic diet for 9 weeks. As shown in Fig. 7C, the plasma ATP increased significantly in atherosclerotic $\mathrm{LDLR}^{-/-}$mice while the $\mathrm{GsdmD}^{-/-} \mathrm{LDLR}^{-/-}$double knockout (KO) mice showed significant attenuation in levels of plasma ATP. These data indicate the decreased IL-1 $\beta$ release in $\mathrm{GsdmD}^{-/-} \mathrm{LDLR}^{-/-}$double $\mathrm{KO}$ mice can decrease foam cell membrane disintegration and reduce ATP release in plasma.

\section{Discussion}

Chronic inflammation is a key player in promoting atherosclerosis and cardiovascular disease (32). The link between cholesterol efflux by ABC transporters, inflammatory cytokines, and heart disease is becoming clearer with studies showing that inflammation can promote atherosclerosis by dampening the RCT pathway $(15,33)$. Many studies have highlighted the role of NIrp3 inflammasome/IL-1 $\beta$ pathway in promoting CVD $(1,3,4,25,34)$ and the link between inflammation and CVD is further highlighted by the link between sepsis survivor and higher risk of having a heart attack or stroke (35). The role of GsdmD in sterile inflammatory diseases, such as atherosclerosis, is not yet clear. We found that in-vivo NIrp3 inflammasome assembly in mice by LPS+ATP injections leads to GsdmD -dependent increase in IL-1 $\beta$ levels and reduced reverse cholesterol transport (Fig. 1, 4). The macrophages lacking GsdmD were more resistant to inflammation-induced defects in cholesterol efflux (Fig. 2). The induction of Nlrp3 inflammasome and ensuing membrane damage due to pore generation by GsdmD can reduce the cell's ability to efficiently transport cholesterol via the ABCA1-apoA1 pathway. To circumvent the membrane damage contribution towards reduced cholesterol efflux, we used conditioned media isolated from WT BMDMs treated with LPS+ATP to show can reduction in cholesterol efflux. These data pointed toward a more direct role of IL-1 $\beta$ in inhibiting cholesterol efflux. Binding of IL-1 $\beta$ to IL-1 receptor (IL-1r) may activate or block cellular 
signaling pathways involved in apoA1-ABCA1 cholesterol efflux pathway. The identity of these signaling pathways remains to be elucidated. Another possibility is the disruption of PIP2 trafficking in WT macrophages by the NIrp3 inflammasome. ABCA1 was identified as the first PIP2 floppase, translocating PIP2 from the inner to outer leaflet of the plasma membrane (27). The ABC transporter mediated PIP2 flop was later confirmed by other studies $(28,36)$. We have shown before that treatment of macrophages with FDA approved antileishmanial drugs Miltefosine can cause PIP2 mislocalization from the inner leaflet of plasma membrane (37). Miltefosine treated macrophages showed defective NIrp3 inflammasome assembly and reduced IL-1 $\beta$ release (37). Cleaved GsdmD and cleaved IL-1 $\beta$ were reported to bind PIP2 directly, and this binding may retain PIP2 on the inner leaflet and thus reduce PIP2 on the cell surface in the WT macrophages. The GsdmD $\mathrm{G}^{-/-}$macrophages on the other hand may retain the ability to translocate PIP2 to the cell surface, and thus sustain more cholesterol efflux activity. In support of role of PIP2 in modulating pyroptosis, the cells expressing ABCA1 or PIP2 binding pleckstrin homology domain showed reduced GsdmD-NT mediated cell lysis (Fig. 2F, 2G).

GsdmD ${ }^{-/-}$mice showed significantly reduced atherosclerosis in LDLr ASO generated hyperlipidemic mice, indicating that GsdmD promotes atherosclerosis in hyperlipidemic mice (Fig. 6). Recent studies in apoE ${ }^{-/-} \mathrm{KO}$ mice using chemical or adenovirus associated inhibition of GsdmD expression also showed a reduction in atherosclerosis $(38,39)$. GsdmD may affect atherosclerotic plaque progression via several different mechanisms. One of the mechanisms could a simple blockage of IL-1 $\beta$ and IL-18 release from inflamed immune cells. This mechanism is supported by the outcome of CANTOS trial showing that anti-IL-1 $\beta$ antibodies reduced MACE in CVD patients (4) and by a previous study showing the role of IL-18 in atherosclerosis in mice (40). Another mechanism by which GsdmD can promote 
atherosclerosis could be via regulating pyroptosis (18-20). Unlike apoptosis, which has been a main focus of studies aimed at understanding macrophage cell death in plaque area due to cholesterol overload, the role of pyroptotic cell death in atherosclerosis is not clear. The presence of cleaved N-terminal fragment of GsdmD in aortic tissue from atherosclerotic plaque-bearing mice may indicate the role of pyroptotic cell death in the progression of atherosclerosis, but is not a conclusive evidence as GsdmD can also get cleaved in living macrophages (7). The clearance of apoptotic cells by phagocytosis in plaque is of high importance as defective efferocytosis promotes further inflammation and plaque progression (41). The pyroptotic cells may not be cleared by efferocytosis as effectively as apoptotic cells due to the lack of proper "eat-me signal", which is an exposure of phosphatidylserine (PS) on the cell surface. Importantly, a recent study has shown the existence of a robust apoptotic caspase network that is activated in parallel to GsdmD-mediated plasma membrane permeabilization and shifts the balance between apoptotic and pyroptotic macrophage cell death (42). We found that lipid-laden GsdmD KO foam cells exhibit higher apoptotic cell death vs. WT macrophages (Fig. 3C). Thus, $\mathrm{KO}$ of GsdmD may reduce atherogenic pyroptotic cell death, and may tilt the balance toward athero-protective apoptotic cell death.

Neutrophil extracellular traps (NETs) play important role in trapping extracellular pathogens but they can promote atherosclerosis by activating macrophages to release more pro-inflammatory cytokines. Hyperlipidemia and cholesterol crystals can promote the formation of NETs that in turn can play role in the progression of atherosclerosis $(43,44)$. Interestingly, $\mathrm{GsdmD}$ is also involved in NETosis and is proteolytically activated by Neutrophil proteases (5). Thus, GsdmD $\mathrm{KO}$ may dampen the progression of atherosclerosis due to reduced formation of NETs. Further studies are required to decipher if the GsdmD atherogenic activity is mediated solely by macrophages or other immune cells such as neutrophils, B-cells, or T-cells, also contribute to 
GsdmD atherogenic activity. Recently, AIM2 inflammasome, caspase 1 and 11, and GsdmD were shown to play in role in clonal haematopoiesis mediated exacerbation of atherosclerosis(45). Clonal haematopoiesis increases the risk of myocardial infarction and stroke independently of traditional risk factors $(46,47)$. The JAK2V617F (JAK2VF) mutation leads to clonal haematopoiesis via increased JAK-STAT signaling, Using mice that express Jak2VF selectively in macrophages, Gsdmd deletion was shown to reduce necrotic core formation and increase cap thickness, but showed no change in overall lesion area (45). Similarly, the bone-marrow transplanted $\mathrm{Gsdmd}^{-/-}$into $\mathrm{Ldlr}^{-/-}$control mice did not change the lesion area (45). These data and our study indicate that GsdmD may also have a non-myeloid role in promoting atherosclerosis. Previous studies have shown the major role of vascular smooth muscle cells (VSMCs) in promoting atherosclerosis, with data showing that VSMCs can adopt phenotypes resembling foam cells and macrophages(48-50). The whole-body knockout of GsdmD may alter the response of VSMCs to lipids and other inflammatory stimuli to modulate progression of atherosclerosis. Similarly, the GsdmD KO may affect adaptive responses of endothelial cells, including but not limited to autophagy and pyroptosis, to lipidburden and cytokines released from inflamed foam cells.

Our data show a novel role of GsdmD in the progression of atherosclerosis, independent of lipid levels. The idea of combining lipid-lowering therapeutics with anti-inflammatory drugs is getting increasingly recognized (51), and targeting GsdmD in combination of LDL lowering therapies such as statins or PCSK9 antibody may serve as potential therapeutic to treat atherosclerosis and cardiovascular disease.

Highlights of this work: The NIrp3/AIM2 inflammasome target protein "GsdmD" is involved in multiple pro-inflammatory pathways such as the release of IL-1 $\beta$, pyroptotic cell death, and 
NETosis. Our data provide the first evidence for the role of GsdmD in promoting atherosclerosis. Thus, targeting GsdmD in conjunction with current LDL lowering therapies may provide additional benefits compared to anti-IL-1 $\beta$ therapy, via mechanisms of decreasing pyroptosis and NETosis.

- Macrophages lacking GsdmD are more resistant to defects in cholesterol efflux after inflammasome activation.

- Treatment of macrophages with conditioned media isolated from inflamed WT, but not the $\mathrm{GsdmD}^{-/-}$macrophages, decreased apoA1 binding to the cells.

- $\mathrm{ABCA} 1$ expression reduced the binding of $\mathrm{N}$-terminal fragment of $\mathrm{GsdmD}$ to the cell membrane and prevented cell lysis. These effects are attributed to ABCA1's PIP2 floppase activity.

- The conditioned media from inflammasome-activated WT, but not the $\mathrm{GsdmD}^{-/-}$ macrophages, promotes foam cell formation.

- Inflammasome-activated GsdmD ${ }^{-/-}$macrophages are more susceptible to apoptotic cell death.

- $\mathrm{GsdmD}$-/- mice show blunted suppression of RCT in vivo (plasma, liver, feces) upon inflammasome activation.

- GsdmD is cleaved in aortic root plaques and LDLR ASO treated GsdmD ${ }^{-/-}$mice show reduced VCAM1 expression in aortic root plaques and decreased atherosclerotic lesions.

\section{Material and Methods}

Mice and diets: All animal experiments were performed in accordance with protocols approved by the Cleveland State University and Cleveland Clinic Institutional Animal Care and 
Use Committee. The C57BL6J mice were purchased from the Jackson Laboratory and the GsdmD ${ }^{-/-}$KO-C57BL/6J mice were generated earlier (52) and kindly provided by Dr. Russell Vance (UC, Berkeley). The GsdmD ${ }^{-/-} \mathrm{KO}$ genotype was confirmed by PCR \& sequencing of Exon 2 of GsdmD using primers, GsdmD Fwd: ATAGAACCC GTGGAGTCCCA and GsdmD Rev: GGCTTCCCTCATTCAGTGCT as described earlier (52). The 12-week-old mice, males and females, in $\mathrm{C} 57 \mathrm{BL} / 6 \mathrm{~J}$ or $\mathrm{GsdmD}^{-/-}$C57BL/6J backgrounds were maintained in a temperature-controlled facility with a 12-h light/dark cycle. Mice were given free access to food and water. The standard chow diet (SD, 20\% kcal protein, 70\% kcal carbohydrate and $10 \%$ kcal fat, Harlan Teklad) was used for regular maintenance and breeding. For generating hyperlipidemia for atherosclerosis studies, mice were fed an atherogenic Western type diet (WD) (ENVIGO, 0.2\% cholesterol with 42\% adjusted calories from fat, TD.88137).

ASO injections: GalNAc-conjugated Gen 2.5 ASO targeting mouse Low-density Lipoprotein receptor (LDLr) and control ASO were kindly provided by Adam Mullick from Ionis Pharmaceuticals. The. ASO treatment started at week 12 with i.p. injection of $5 \mathrm{mg} / \mathrm{Kg}$ body weight, 1 x per week until sacrifice at week 21 . The oligo stock was prepared at $500 \mathrm{mg} / \mathrm{ml}$ in sterile saline, such that a $25 \mathrm{~g}$ mouse would receive a $250 \mu$ injection. The control group receive 1x per week control ASO. Following the ASO injection for 9 weeks, animals were sacrificed and hearts were perfused for sectioning for quantification of atherosclerotic lesions. Isolation of Bone marrow derived macrophages: WT C57BL/6 or C57BL/6 GsdmD ${ }^{-/-} \mathrm{KO}$ mice were maintained on standard chow diet and water. Mice were euthanized by $\mathrm{CO}_{2}$ inhalation and femoral bones were removed and flushed for marrow for isolating bone-marrow cells Detailed method for preparing bone-marrow derived macrophages is described in Supplementary material and methods section. 
In-vivo NIrp3 inflammasome assembly and IL-1 $\beta$ release assays: The 12-week-old WT or GsdmD ${ }^{-/-}$mice were IP injected with either $5 \mu \mathrm{g}$ LPS, or sterile PBS. After 4h of LPS or PBS injection, the NIrp3 inflammasome assembly in mice was induced by IP injection of ATP (0.5 $\mathrm{ml}$ of $30 \mathrm{mM}, \mathrm{pH} 7.0)$. The mice were euthanized after $30 \mathrm{~min}$ and peritoneal cavity was lavaged with $5 \mathrm{ml}$ PBS. Approximately $3.5 \mathrm{ml}$ peritoneal lavage fluid was recovered from each mouse and centrifuged at $15 \mathrm{~K}$ for $10 \mathrm{~min}$ at room temperature. The supernatant was subjected to IL-1 $\beta$ ELISA, using mouse IL-1b Quantikine ELISA kit (MLB00C, R \& D systems) and following manufacturer's instructions.

Cholesterol efflux assay: Cholesterol efflux assays were performed as described earlier (27). The WT or GsdmD ${ }^{-/-}$BMDMs were radiolabeled with $0.5 \mu \mathrm{Ci} / \mathrm{ml}$ of $[3 \mathrm{H}]$-cholesterol, ABCA1 expression in BMDMs was induced by treatment with T0901317, and chase was performed with serum free media containing cholesterol acceptor apoA1. The percent cholesterol efflux was calculated as $100 \times($ medium dpm) / (medium dpm + cell dpm). Detailed method is described in Supplementary material and methods section.

Mice RCT assay. WT Murine bone marrow derived macrophages were incubated with tritium labeled $[3 \mathrm{H}]$ cholesterol and acetylated LDL to generate foam cells. The day prior to radiolabeled foam cell injection, mice received intraperitoneal injections of PBS or $5 \mu \mathrm{g} / \mathrm{ml}$ LPS and three hours later mice treated with LPS received $30 \mathrm{mM}$ of adenosine tri-phosphate, (sigma) $\mathrm{pH}=7.5-8$. Mice received daily LPS/ATP injection through the course of the study. RCT to the plasma, liver, and feces was calculated as the \% (dpm appearing in plasma/total dpm injected). Detailed method is described in Supplementary material and methods section. Western blotting: BMDMs or RAW264.7 cells were grown and treated as indicated. The PBSwashed cell pellet was lysed in MPER or RIPA lysis buffer supplemented with protease 
inhibitors and PMSF. Detailed method is described in Supplementary material and methods section.

Cholesterol measurements: Total cholesterol was measured by using Stan Bio Total cholesterol reagent (\#1010-225), following manufacturer's instructions.

Atherosclerotic lesion quantification: Mice were sacrificed by $\mathrm{CO}_{2}$ inhalation and weighed at 21 weeks of age. Whole blood was collected from the retroorbital plexus into a heparinized glass capillary, mixed with EDTA and spun in a microfuge to obtain plasma. The circulatory system was perfused with $10 \mathrm{~mL}$ PBS and the heart was excised and preserved in $10 \%$ phosphate buffered formalin. The quantitative assessment of atherosclerotic plaque area in the aortic root was performed as previously described (53). Lesion areas were quantified as the mean value in multiple sections at $80 \mu \mathrm{m}$ intervals using Image Pro software (Media Cybernetics).

Statistics. All statistics were performed using GraphPad Prism software. Comparison of two groups was performed by two-tailed $t$-test and comparison of more than two groups was performed by ANOVA with the specified posttest. Values shown are mean \pm SD.

\section{References}

1. P. Duewell et al., NLRP3 inflammasomes are required for atherogenesis and activated by cholesterol crystals. Nature 464, 1357-1361 ( 2010 ).

2. B. Vandanmagsar et al., The NLRP3 inflammasome instigates obesity-induced inflammation and insulin resistance. Nat Med 17, 179_188 (2011).

3. A. Janoudi, F. E. Shamoun, J. K. Kalavakunta, G. S. Abela, Cholesterol crystal induced arterial inflammation and destabilization of atherosclerotic plaque. Eur Heart J 37, 1959_ 1967 (2016).

4. P. M. Ridker et al., Antiinflammatory Therapy with Canakinumab for Atherosclerotic Disease. N Engl J Med 377, 1119_1131 (2017).

5. G. Sollberger et al., Gasdermin D plays a vital role in the generation of neutrophil extracellular traps. Sci Immunol 3, (2018). 
6. J. Lieberman, H. Wu, J. C. Kagan, Gasdermin D activity in inflammation and host defense. Sci Immunol 4, (2019).

7. C. L. Evavold et al., The Pore_Forming Protein Gasdermin D Regulates Interleukin_1 Secretion from Living Macrophages. Immunity 48, 35_44 e36 (2018).

8. T. Bergsbaken, S. L. Fink, B. T. Cookson, Pyroptosis: host cell death and inflammation. Nature reviews. Microbiology 7, 99-109 (2009).

9. S. Ruhl et al., ESCRT_dependent membrane repair negatively regulates pyroptosis downstream of GSDMD activation. Science 362, 956-960 ( 2018 ).

10. K. J. Moore, I. Tabas, Macrophages in the pathogenesis of atherosclerosis. Cell 145, 341_ 355 ( 2011).

11. P. Libby, P. M. Ridker, A. Maseri, Inflammation and atherosclerosis. Circulation 105, 1135_ 1143 (2002).

12. A. R. Tall, L. Yvan_Charvet, Cholesterol, inflammation and innate immunity. Nat Rev Immunol 15, 104-116 (2015).

13. A. V. Khera et al., Cholesterol efflux capacity, high_density lipoprotein function, and atherosclerosis. The New England journal of medicine 364, 127-135 (2011).

14. L. Yvan_Charvet, N. Wang, A. R. Tall, Role of HDL, ABCA1, and ABCG1 transporters in cholesterol efflux and immune responses. Arterioscler Thromb Vasc Biol 30, 139_143 (2010).

15. F. C. McGillicuddy et al., Inflammation impairs reverse cholesterol transport in vivo. Circulation 119, 1135-1145 (2009).

16. P. Malik et al., Zymosan-mediated inflammation impairs in vivo reverse cholesterol transport. J Lipid Res 52, 951_957 (2011).

17. B. Razani et al., Autophagy links inflammasomes to atherosclerotic progression. Cell Metab 15, 534-544 (2012).

18. X. Liu et al., Inflammasome-activated gasdermin D causes pyroptosis by forming membrane pores. Nature 535, 153-158 (2016).

19. J. Ding et al., Pore-forming activity and structural autoinhibition of the gasdermin family. Nature 535, 111-116 (2016).

20. N. Kayagaki et al., Caspase_11 cleaves gasdermin D for non_canonical inflammasome signalling. Nature 526, 666-671 (2015).

21. M. Monteleone et al., Interleukin-1beta Maturation Triggers Its Relocation to the Plasma Membrane for Gasdermin_D_Dependent and _Independent Secretion. Cell Rep 24, 1425_ 1433 (2018).

22. C. L. Evavold et al., The Pore_Forming Protein Gasdermin D Regulates Interleukin_1 Secretion from Living Macrophages. Immunity, (2017). 
23. B. Feng et al., The endoplasmic reticulum is the site of cholesterol_induced cytotoxicity in macrophages. Nature cell biology 5, 781-792 (2003).

24. X. Liao et al., Macrophage autophagy plays a protective role in advanced atherosclerosis. Cell Metab 15, 545-553 (2012).

25. K. Rajamaki et al., Cholesterol crystals activate the NLRP3 inflammasome in human macrophages: a novel link between cholesterol metabolism and inflammation. PLoS One 5, e11765 (2010).

26. S. Wang, K. Gulshan, G. Brubaker, S. L. Hazen, J. D. Smith, ABCA1 mediates unfolding of apolipoprotein AI N terminus on the cell surface before lipidation and release of nascent high_density lipoprotein. Arterioscler Thromb Vasc Biol 33, 1197_1205 ( 2013 ).

27. K. Gulshan et al. , PI (4,5)P2 Is Translocated by ABCA1 to the Cell Surface Where It Mediates Apolipoprotein A1 Binding and Nascent HDL Assembly. Circ Res 119, 827_838 ( 2016 ).

28. O. Vivas, S. A. Tiscione, R. E. Dixon, D. S. Ory, E. J. Dickson, Niemann_Pick Type C Disease Reveals a Link between Lysosomal Cholesterol and PtdIns (4,5)P2 That Regulates Neuronal Excitability. Cell Rep 27, 2636-2648 e2634 (2019).

29. S. Baghirova, B. G. Hughes, M. J. Hendzel, R. Schulz, Sequential fractionation and isolation of subcellular proteins from tissue or cultured cells. MethodsX 2, 440_445 ( 2015 ).

30. D. Basu et al., Novel Reversible Model of Atherosclerosis and Regression Using Oligonucleotide Regulation of the LDL Receptor. Circ Res 122, 560-567 ( 2018 ).

31. P. Stachon et al., Extracellular ATP Induces Vascular Inflammation and Atherosclerosis via Purinergic Receptor Y2 in Mice. Arterioscler Thromb Vasc Biol 36, 1577_1586 (2016).

32. S. E. Nissen et al., Effect of very high-intensity statin therapy on regression of coronary atherosclerosis: the ASTEROID trial. JAMA 295, 1556-1565 (2006).

33. L. Yvan_Charvet et al., Combined deficiency of ABCA1 and ABCG1 promotes foam cell accumulation and accelerates atherosclerosis in mice. The Journal of clinical investigation 117, 3900_3908 (2007).

34. T. van der Heijden et al., NLRP3 Inflammasome Inhibition by MCC950 Reduces Atherosclerotic Lesion Development in Apolipoprotein E_Deficient Mice_Brief Report. Arterioscler Thromb Vasc Biol 37, 1457-1461 ( 2017 ).

35. C. C. Lai et al., Susceptible period for cardiovascular complications in patients recovering from sepsis. CMAJ 190, E1062_E1069 (2018).

36. J. P. Deppe et al., The wheat ABC transporter Lr34 modifies the lipid environment at the plasma membrane. The Journal of biological chemistry 293, 18667-18679 (2018).

37. A. J. Iacano et al., Miltefosine increases macrophage cholesterol release and inhibits NLRP3_inflammasome assembly and IL_1beta release. Sci Rep 9, 11128 ( 2019 ).

38. S. S. Xing et al., Salidroside Decreases Atherosclerosis Plaque Formation via Inhibiting Endothelial Cell Pyroptosis. Inflammation 43, 433_440 ( 2020 ). 
39. M. Jiang et al., Caspase_11_Gasdermin D_Mediated Pyroptosis Is Involved in the Pathogenesis of Atherosclerosis. Front Pharmacol 12, 657486 (2021).

40. S. C. Whitman, P. Ravisankar, A. Daugherty, Interleukin_18 enhances atherosclerosis in apolipoprotein $\mathrm{E}_{\left(-I_{-}\right)}$mice through release of interferon-gamma. Circ Res 90, E34_38 (2002).

41. C. Kasikara, A. C. Doran, B. Cai, I. Tabas, The role of non-resolving inflammation in atherosclerosis. J Clin Invest 128, 2713-2723 (2018).

42. N. M. de Vasconcelos et al., An Apoptotic Caspase Network Safeguards Cell Death Induction in Pyroptotic Macrophages. Cell Rep 32, 107959 (2020).

43. A. Warnatsch, M. Ioannou, Q. Wang, V. Papayannopoulos, Inflammation. Neutrophil extracellular traps license macrophages for cytokine production in atherosclerosis. Science 349, 316-320 (2015).

44. Y. Doring, P. Libby, O. Soehnlein, Neutrophil Extracellular Traps Participate in Cardiovascular Diseases: Recent Experimental and Clinical Insights. Circ Res 126, 1228_ 1241 (2020).

45. T. P. Fidler et al., The AIM2 inflammasome exacerbates atherosclerosis in clonal haematopoiesis. Nature 592, 296-301 (2021).

46. S. Jaiswal et al., Clonal Hematopoiesis and Risk of Atherosclerotic Cardiovascular Disease. The New England journal of medicine 377, 111-121 (2017).

47. A. G. Bick et al., Author Correction: Inherited causes of clonal haematopoiesis in 97,691 whole genomes. Nature 591, E27 (2021).

48. G. L. Basatemur, H. F. Jorgensen, M. C. H. Clarke, M. R. Bennett, Z. Mallat, Vascular smooth muscle cells in atherosclerosis. Nature reviews. Cardiology 16, 727_744 (2019).

49. T. Kunieda et al., Angiotensin II induces premature senescence of vascular smooth muscle cells and accelerates the development of atherosclerosis via a p21_dependent pathway. Circulation 114, 953-960 (2006).

50. M. C. Clarke et al., Apoptosis of vascular smooth muscle cells induces features of plaque vulnerability in atherosclerosis. Nature medicine 12, 1075-1080 (2006).

51. P. M. Ridker, From CANTOS to CIRT to COLCOT to Clinic: Will All Atherosclerosis Patients Soon Be Treated With Combination Lipid_Lowering and Inflammation_Inhibiting Agents? Circulation 141, 787_789 (2020).

52. I. Rauch et al., NAIP_NLRC4 Inflammasomes Coordinate Intestinal Epithelial Cell Expulsion with Eicosanoid and IL_18 Release via Activation of Caspase_1 and _8. Immunity 46, 649_ 659 (2017).

53. J. Baglione, J. D. Smith, Quantitative assay for mouse atherosclerosis in the aortic root. Methods Mol Med 129, 83-95 (2006). 


\section{Author Contributions}

K.G. designed and directed research. E.O, C.A.T, D.Z, A.J.I, J.H, M.K. and K.G performed research. E.O, C.A.T, and K.G. analyzed data. K.G. drafted the manuscript. All authors critically reviewed the manuscript.

\section{Acknowledgments}

This work was supported by National Institutes of Health Grant RO1HL148158 (to K.G), American Heart Association Scientist Development Grant SDG25710128 (to K.G), and Cleveland State University startup funds (to K.G.). C.A.T is supported by F31HL134231 and J.D.S is supported by RO1HL128268 and R01 HL130085.

\section{Disclosures None.}




\section{Figure legends:}

Fig. 1. A) GsdmD plays a role in release of mature IL-1 $\beta$ upon in-vivo NIrp3 inflammasome activation. A) BMDMs isolated from WTC57BL/6J or C57BL6/J-GsdmD ${ }^{-/-}$mice were primed by incubation with $\pm 1 \mu \mathrm{g} / \mathrm{ml}$ LPS for $4 \mathrm{~h}$. The NIrp3 inflammasome assembly was induced either by incubation with $1 \mathrm{mM}$ ATP for $20 \mathrm{~min}$ or $1 \mu \mathrm{M}$ Nigericin for $1 \mathrm{~h}$. The IL-1 $\beta$ levels in the cell-free media were measured by ELISA (mean $\pm S D, N=6,{ }^{* * *}$ represent $p<0.001$ by ANOVA with Bonferroni posttest). B) WT or GsdmD ${ }^{-/-}$BMDMs were treated $\pm 1 \mu \mathrm{g} / \mathrm{ml}$ LPS, followed by western blot analysis using NIrp3 and-IL-1 $\beta$ antibodies. The $\beta$-actin was used as a loading control. C) The age-matched male WTC57BL/6J or $\mathrm{GsdmD}^{-/}$mice were primed with IP injection of LPS $(5 \mu \mathrm{g} / \mathrm{mouse})$. After $4 \mathrm{~h}$ of LPS injection, the NIrp3 inflammasome assembly in mice was induced by IP injection of ATP $(0.5 \mathrm{ml}$ of $30 \mathrm{mM}, \mathrm{pH} 7.0)$. The peritoneal cavity was lavaged with $3 \mathrm{ml}$ PBS, and IL-1 $\beta$ levels in peritoneal lavage were determined by ELISA $\left(N=6,{ }^{* * * *} p<0.0001\right.$ by two-tailed t-test). D) The age-matched male WTC57BL/6J or GsdmD ${ }^{-/-}$mice were treated with \pm IP injection of LPS $(5 \mu \mathrm{g} / \mathrm{mouse})$ and injected with $0.5 \mathrm{ml}$ of saline. The peritoneal cavity was lavaged with $5 \mathrm{ml}$ PBS, and IL-1 $\beta$ levels in peritoneal lavage were determined by ELISA ( $N=4$, n.s.=non-significant by two-tailed t-test).

Fig. 2 GsdmD promotes NIrp3 inflammasome-mediated defects in reverse cholesterol transport. A) BMDMs isolated from WT or $\mathrm{GsdmD}^{-/-}$mice were loaded with ${ }^{3} \mathrm{H}$-cholesterol + $50 \mu \mathrm{g} / \mathrm{ml}$ AcLDL for $24 \mathrm{~h}$ and treated with LXR-agonist T0901317 (Sigma-Aldrich) to induce ABCA1 expression. The NIrp3 inflammasome was induced by LPS+ Nigericin treatment, followed by cholesterol efflux assay using lipid-free apoA1 $(5 \mu \mathrm{g} / \mathrm{ml})$ in serum-free DMEM as chase media for $4 \mathrm{~h}$ at $37^{\circ} \mathrm{C}$. Values are $\%$ cholesterol efflux (mean $\pm S D, N=4$, ${ }^{* * * *}$ represent $p<0.0001,{ }^{* *}$ represent $p<0.01$ by ANOVA Bonferroni posttest). $\boldsymbol{B}$ ) The BMDMs isolated from WT or GsdmD ${ }^{-1-}$ mice were loaded with ${ }^{3} \mathrm{H}$-cholesterol $+50 \mu \mathrm{g} / \mathrm{ml}$ AcLDL for $24 \mathrm{~h}$. The BMDMs, \pm LXR-agonist T0901317, were treated with conditioned media isolated from LPS+ATP treated WT or GsdmD ${ }^{--}$BMDMs for 16h. The cholesterol efflux assay was performed using lipid-free apoA1 $(5 \mu \mathrm{g} / \mathrm{ml})$ in serum-free DMEM as chase media for $4 \mathrm{~h}$ at $37^{\circ} \mathrm{C}$. Values are $\%$ cholesterol efflux (mean $\pm S D, N=4,{ }^{* * * *}$ represent $p<0.0001$, ${ }^{* * *}$ represent $p<0.001$ by ANOVA Bonferroni posttest). C) The flow cytometry analysis showing histogram bar for Alexa-647 labeled apoA1 binding to cells, \pm ABCA1 expression and \pm WT conditioned media treatment $D$ ) The flow cytometry analysis showing quantification of Alexa-647 labeled apoA1 binding to cells \pm ABCA1 expression and \pm . WT or $\mathrm{GsdmD}^{--}$conditioned media treatment (mean $\pm \mathrm{SD}, \mathrm{N}=4$, ${ }^{* * * *}$ represent $p<0.0001,{ }^{* * *}$ represent $p<0.001$ by ANOVA Bonferroni posttest) $\boldsymbol{E}$ ) The HEK293, HEK293-ABCA1-eGFP, or HEK293-2X-PH-PLC-eGFP cells were transfected with plasmids carrying either full-length (FL) GsdmD or N-terminal fragment (NT) of GsdmD. The LDH release in media is plotted in \% values of total cellular $\mathrm{LDH}$ (mean $\pm \mathrm{SD}, \mathrm{N}=4$; different letters show $\mathrm{p}<0.001$, by ANOVA Bonferroni posttest). $\boldsymbol{F}$ ) The control HEK293 and HEK293 cells stably transfected with ABCA1 or PH-PLC were transfected with plasmid carrying Flag tagged GsdmDNT. The transfected cells were fractionated into cytoplasmic or membrane bound fractions. The fractions were resolved using SDS gel, transferred to nitrocellulose membrane, and probed with antibody against Flag tag.

Fig. 3 GsdmD promotes foam cell formation. A) BMDMs isolated from WT or GsdmD ${ }^{-/-}$mice were loaded with $50 \mu \mathrm{g} / \mathrm{ml}$ AcLDL $+25 \mu \mathrm{g} / \mathrm{ml}$ OxLDL for $48 \mathrm{~h} \pm$ conditioned media isolated from LPS/ATP treated WT or GsdmD/- BMDMs. The culture media was removed, cells were washed 
with PBS, and stained with Nile-red dye $(0.25 \mu \mathrm{g} / \mathrm{ml})$ at $37^{\circ} \mathrm{C}$ for $15 \mathrm{~min}$. The stained cells were washed thoroughly with PBS and fixed with paraformaldehyde and imaged using fluorescent microscope with TRITC filter (Excitation / emission: 550 / $640 \mathrm{~nm}$ ). B) Quantification of Nile-red staining in WT or GsdmD ${ }^{-/-}$BMDMs \pm conditioned media by flow-cytometry, mean $\pm S D, N=4$, ${ }^{* * * *}$ represent $\mathrm{p}<0.0001$ by ANOVA Bonferroni posttest. $\boldsymbol{C}$ ) BMDMs isolated from WT or GsdmDI- mice were loaded with $50 \mu \mathrm{g} / \mathrm{ml} \mathrm{AcLDL}+25 \mu \mathrm{g} / \mathrm{ml}$ OxLDL for $48 \mathrm{~h}$. The foam cells were left untreated or treated with LPS+ATP to induce NIrp3 inflammasome. The cells were washed with PBS, and PS exposure was determined by flow-cytometry based assay using Annexin Cy5 staining (mean $\pm S D, N=4,^{* * * *}$ represent $p<0.0001,{ }^{* * *}$ represent $p<0.001$ by ANOVA Bonferroni posttest, n.s=non-significant).

Fig. 4: GsdmD mediates NIrp3 inflammasome-induced defects in reverse cholesterol transport. A) Schematic representation of mouse RCT study design. The 12-week-old male mice were injected daily with or without 5ug LPS and 30mM ATP for 4 days. On day 2 mice were injected with radiolabeled foam cells loaded with AcLDL and ${ }^{3} \mathrm{H}$ cholesterol. The plasma and feces RCT was assessed at $24,48,72 \mathrm{~h}$ (quantified as a $\%$ of the $24 \mathrm{~h}$ Ctrl for each group) and cumulatively (quantified as a percent of respective Ctrl). B) RCT to plasma in WT mice at 24h, 48h, and 72h. C) RCT to plasma in $\mathrm{GsdmD}^{-/-}$mice at $24 \mathrm{~h}, 48 \mathrm{~h}$, and $72 \mathrm{~h}$. D) Cumulative RCT to plasma in WT and GsdmD ${ }^{-/-}$mice. $\left.\boldsymbol{E}\right)$ RCT to liver in WT and GsdmD ${ }^{-/-}$mice. F) RCT to feces in WT mice at 24h, 48h, and 72h. G) RCT to feces in GsdmD ${ }^{-/-}$mice at 24h, 48h, and $72 \mathrm{~h}$. $\boldsymbol{H})$ Cumulative RCT to feces in WT and $\mathrm{GsdmD}^{-/-}$mice. N=4-5, for WT and GsdmD $\mathrm{Gr}^{-/}$Ctrl mice, $\mathrm{N}=6$-7 for WT and GsdmD ${ }^{-/-}$LPS/ATP mice. ${ }^{* * * *} p<0.0001,{ }^{* * *} p<0.001,{ }^{* *} p<0.01$ by two-tailed ttest. Groups with different letters $a, b, c, d$ is $p<0.05$ by One-way ANOVA.

Fig. 5. LDLr ASO generates hyperlipidemia in both WT and GsdmD Schematic representation of mouse atherosclerosis study design. The 12-week-old mice from both sexes were injected with either control or LDLr ASO for 9 weeks. The mice were fed western type diet throughout the course of study. The body weight in females $(\boldsymbol{B})$ and total plasma cholesterol levels $(\boldsymbol{C})$ at week 0 and at the end of experiment, indicated by week 9 , (mean \pm SD, $\mathrm{N}=12,{ }^{* * * *} \mathrm{p}<0.0001$ by two-tailed t-test, $\mathrm{n} . \mathrm{s}=$ non-significant), and male data for body weight (D) and total plasma cholesterol levels $(\boldsymbol{E})$ is presented at base and final levels $\left(\mathrm{N}=12^{* * * *} \mathrm{p}\right.$ $<0.0001$ by two-tailed t-test for both WT-week 0 vs. WT-week 9 and GsdmD ${ }^{-/-}$-week 0 vs. GsdmD ${ }^{-/-}$-week 9 , n.s= non-significant by two-tailed t-test).

Fig. 6. GsdmD promotes atherosclerosis and is cleaved in atherosclerotic plaques. The 12-weeks old mice from both sexes were injected with either control or LDLr ASO for 9 weeks and were fed western type diet throughout the course of study. Mice were euthanized after 9week ASO treatment and were perfused with PBS and hearts and livers were removed. A) A small portion of liver from mice was excised, weighed, and protein extracts were prepared using RIPA buffer supplemented with protease inhibitors. Western blot analysis of LDLr in liver extracts was performed using LDLr antibody and HRP conjugated secondary antibody. B) Representative images from both sexes showing aortic root lesions stained with Oil red $O$ and hematoxylin from WT or GsdmD ${ }^{--} \mathrm{KO}$ mice injected with LDLr ASO and fed WTD, $\boldsymbol{C}, \boldsymbol{D}$ ) Quantification of aortic root lesions in females and males from WT or GsdmD ${ }^{-/-}$KO mice treated with LDLr ASO and fed WTD (for WT male N=11, for WT females, GsdmD ${ }^{-/-}$males, and GsdmD ${ }^{-/}$females, $N=12,{ }^{* * *} p<0.0005,{ }^{* * * *} p<0.0001$ by two-tailed t-test).E) Protein extracts from aortas of control ASO or LDLr ASO treated mice $(\mathrm{N}=4)$ were subjected to western blot analysis using antibody specific for cleaved GsdmD and VCAM-1. GAPDH was probed as a 
loading control. F) ATP levels in plasma of control ASO or LDLr ASO treated mice fed with atherogenic diet were determined by ATP quantification kit, following manufacturer's instructions. (mean $\pm S D, N=8$; different letters show $p<0.001$, by ANOVA Bonferroni posttest).

Fig. 7. GsdmD is cleaved in atherosclerotic plaques. A) The 12-weeks old mice were injected with either control or LDLr ASO for 9 weeks and were fed western type diet throughout the course of study. Mice were euthanized after 9-week ASO treatment and protein extracts from aortas of control ASO or LDLr ASO treated mice $(\mathrm{N}=4)$ were subjected to western blot analysis using antibody specific for cleaved GsdmD and VCAM-1. GAPDH was probed as a loading control. B) The fresh Frozen hearts were sectioned, followed by antigen retrieval and endogenous peroxidase activity blocking, and probing with antibody specific for cleaved Nterminal fragment of Gasdermin D. C) ATP levels in plasma of control ASO or LDLr ASO treated mice fed with atherogenic diet were determined by ATP quantification kit, following manufacturer's instructions. (mean $\pm S D, N=8$; different letters show $p<0.001$, by ANOVA Bonferroni posttest). 
Figure 1

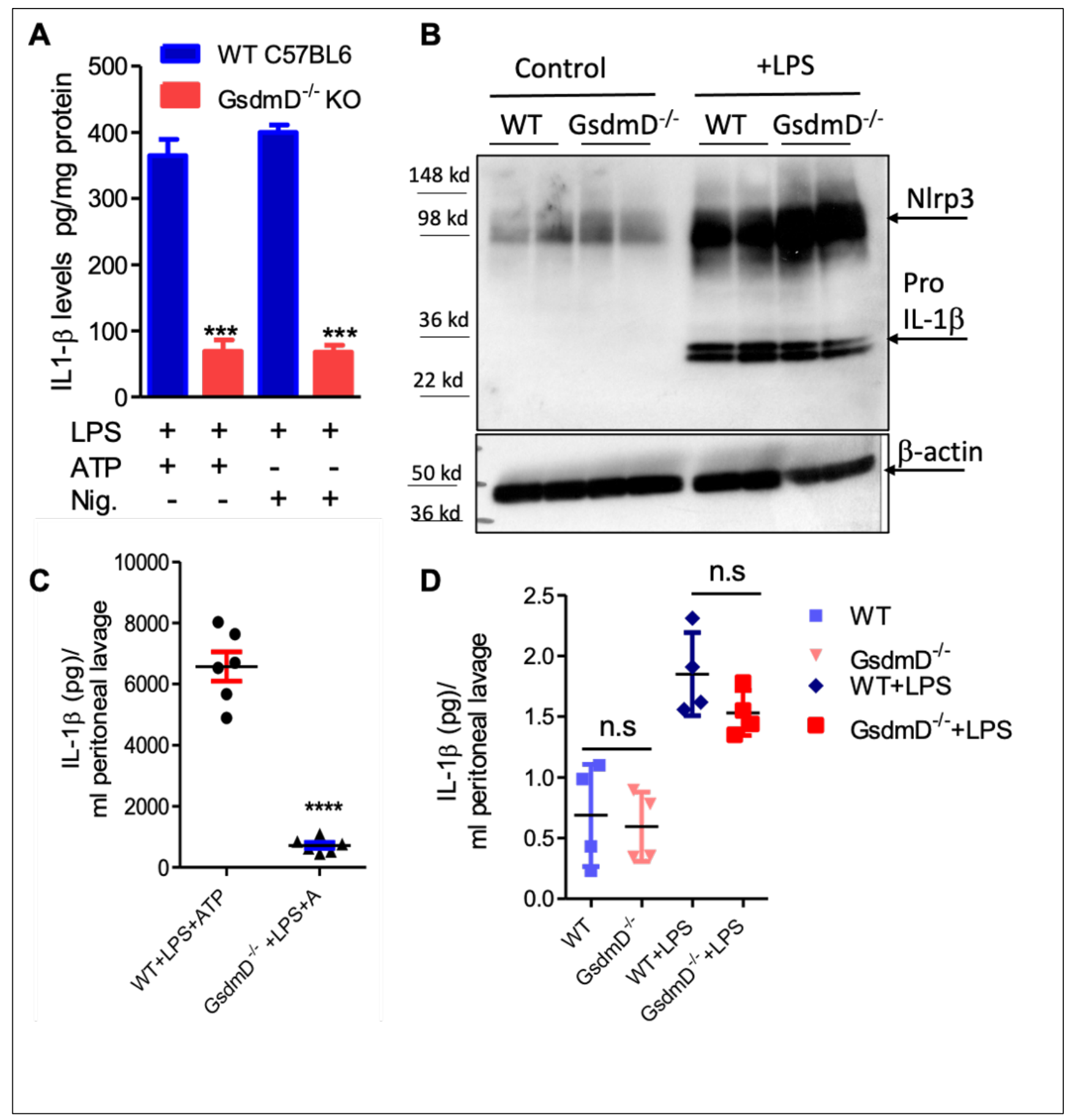


Figure 2

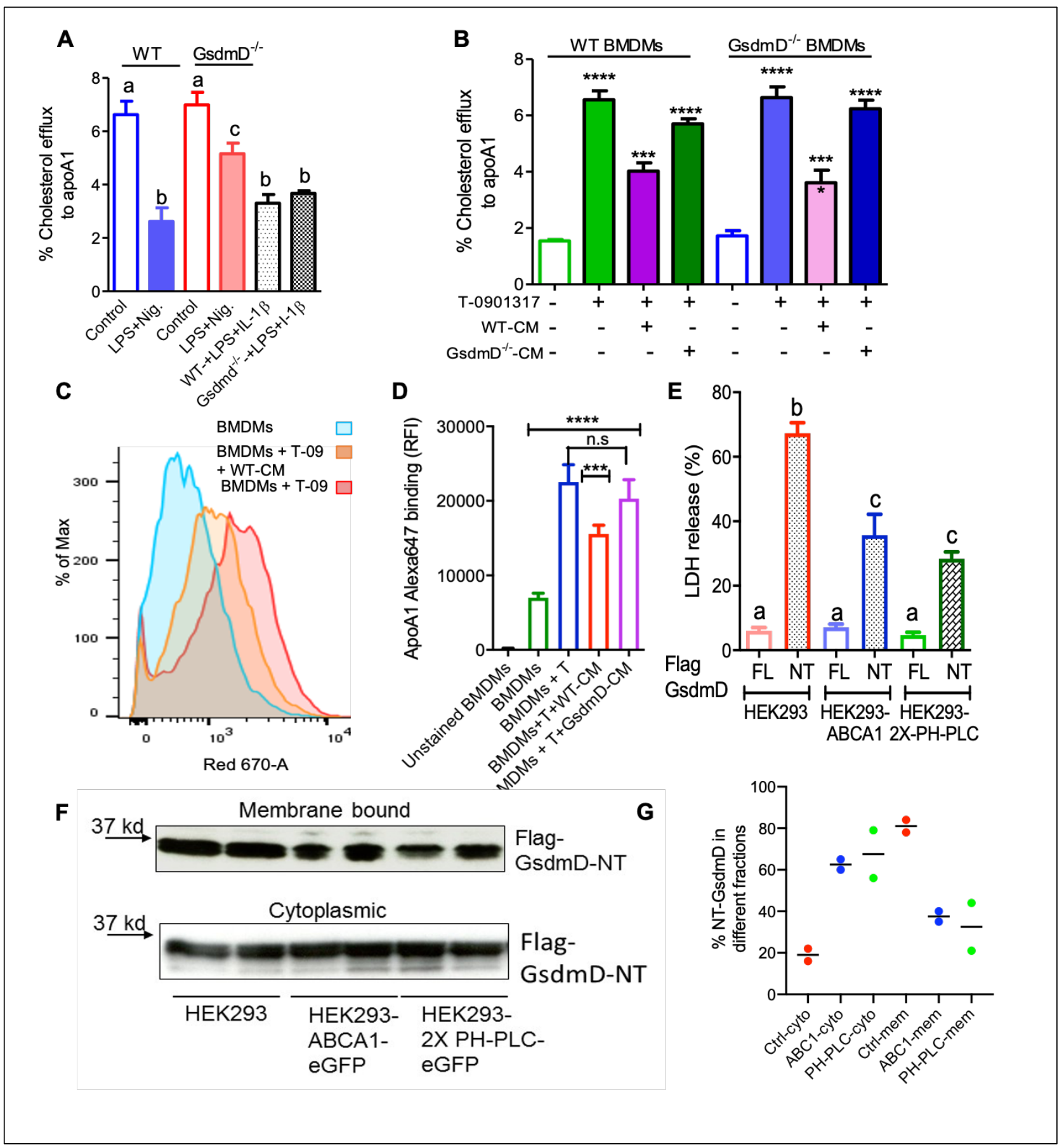


Figure 3

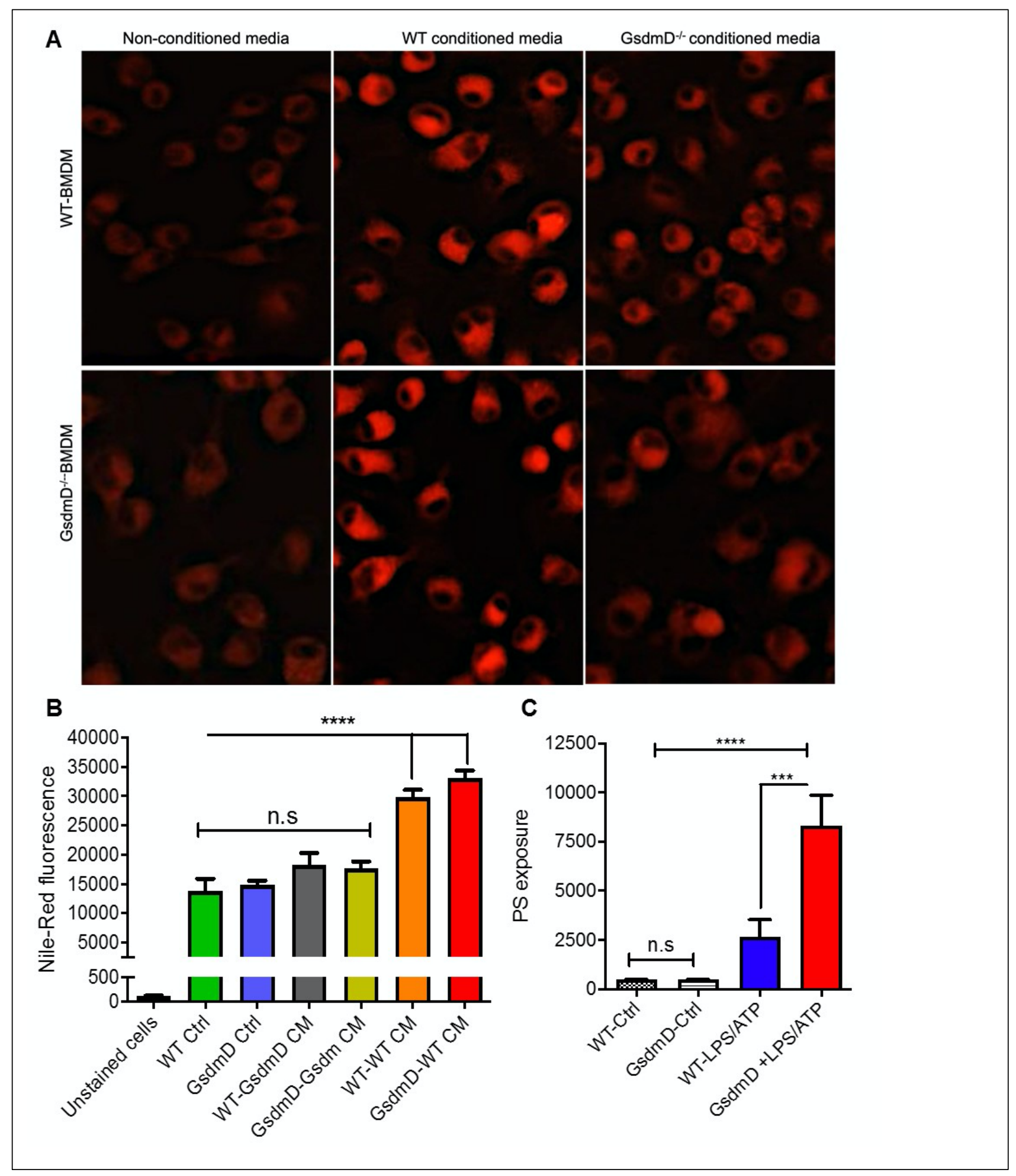




\section{Figure 4}

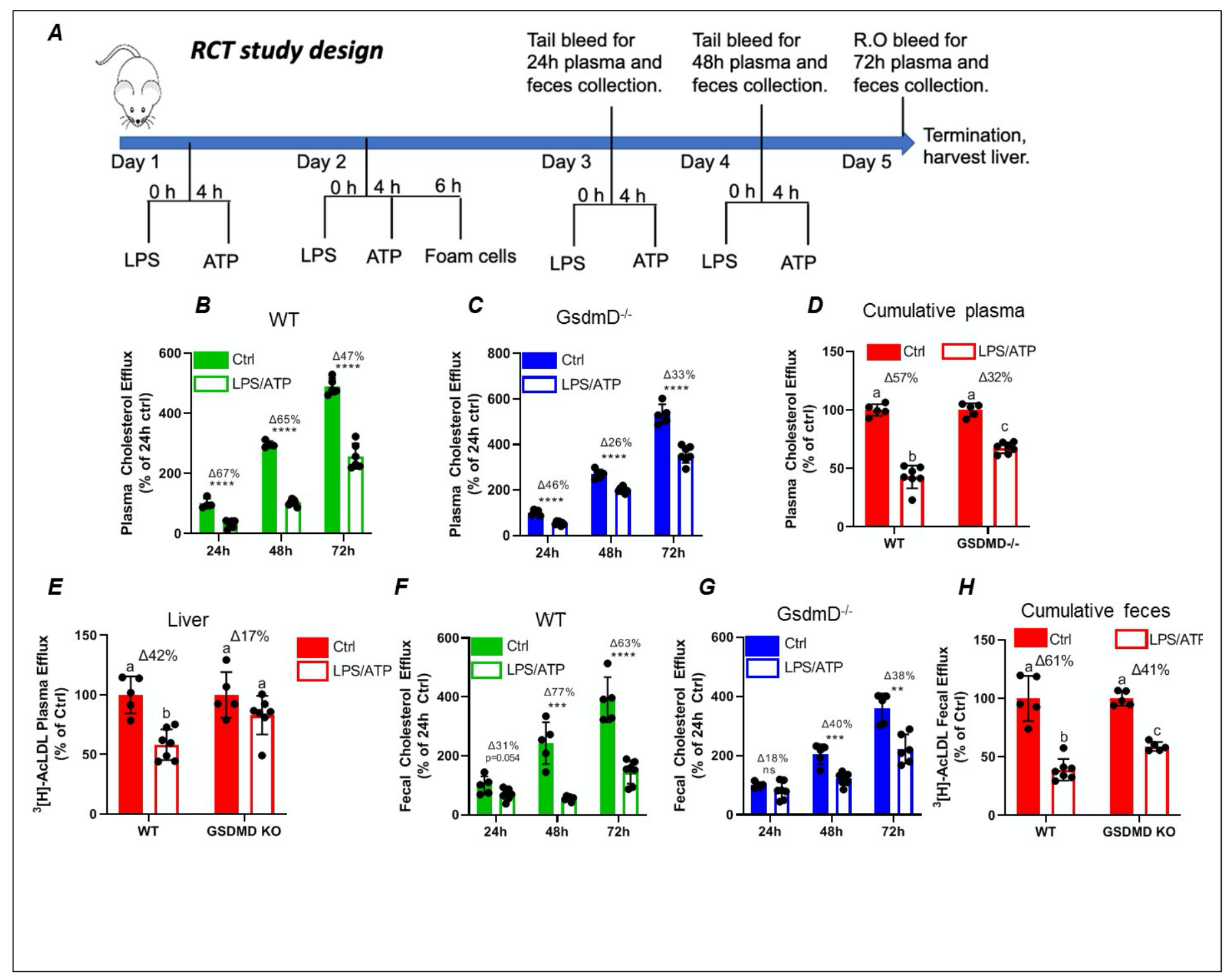


Figure 5

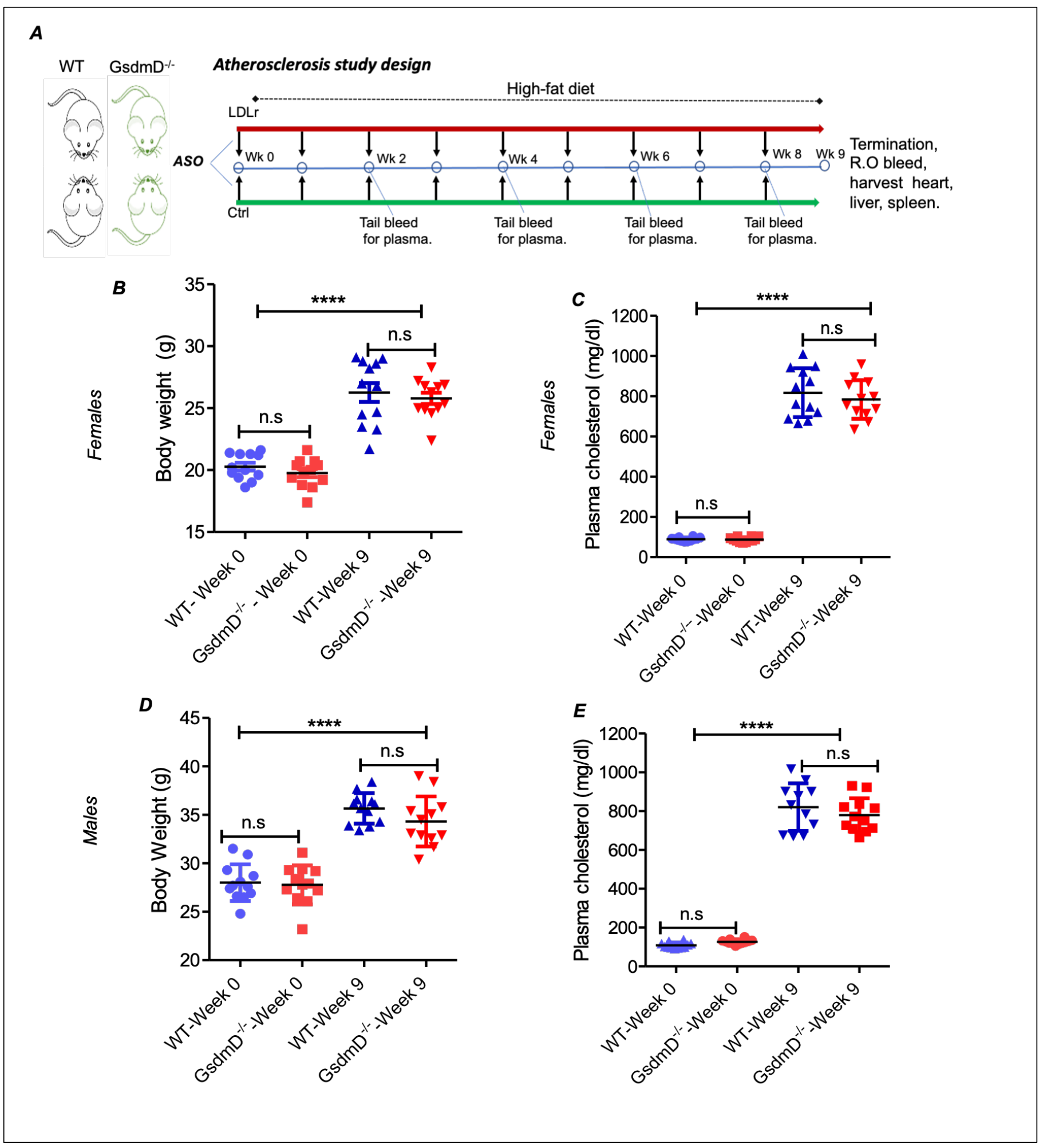


Figure 6

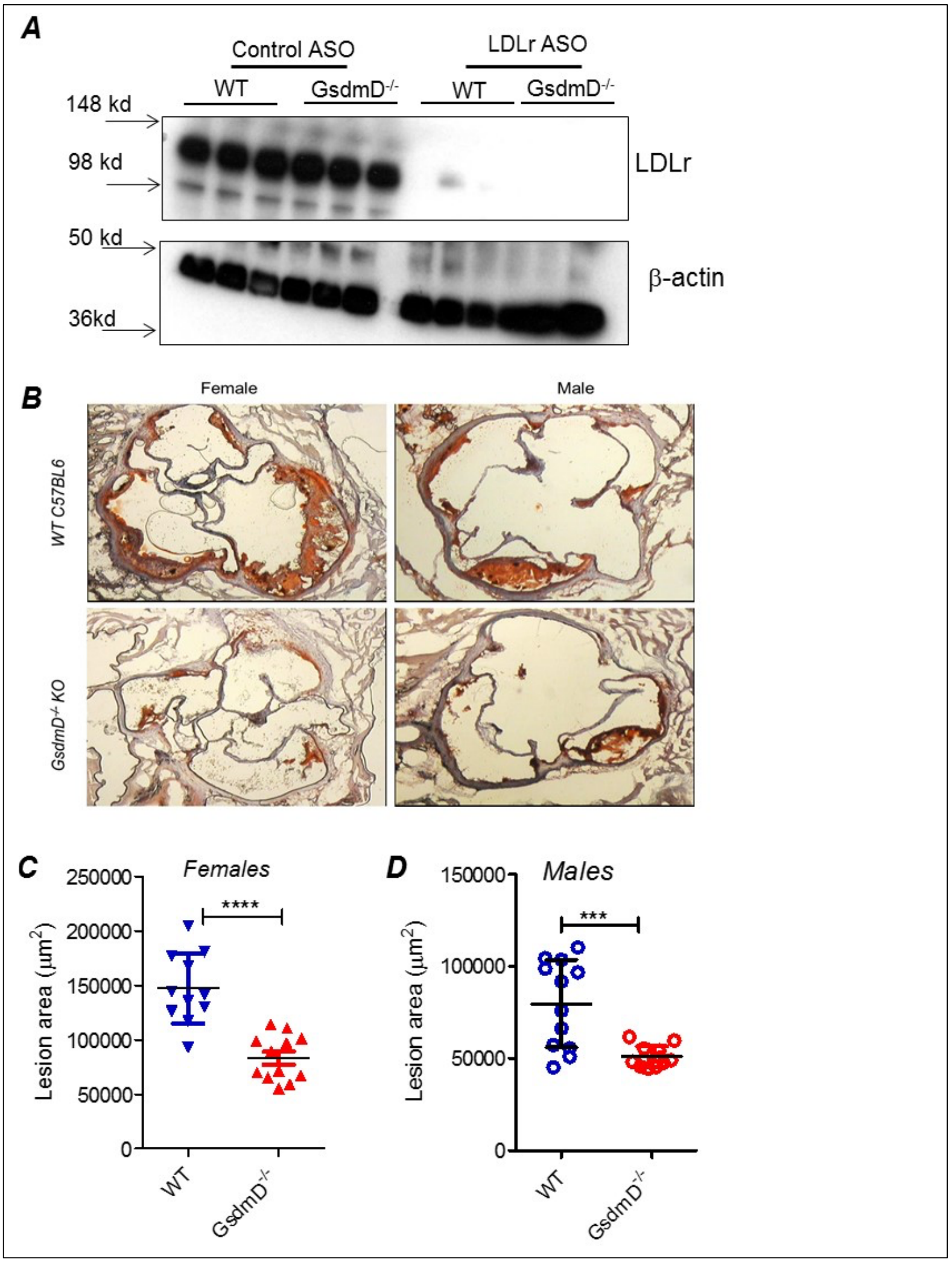


Figure 7

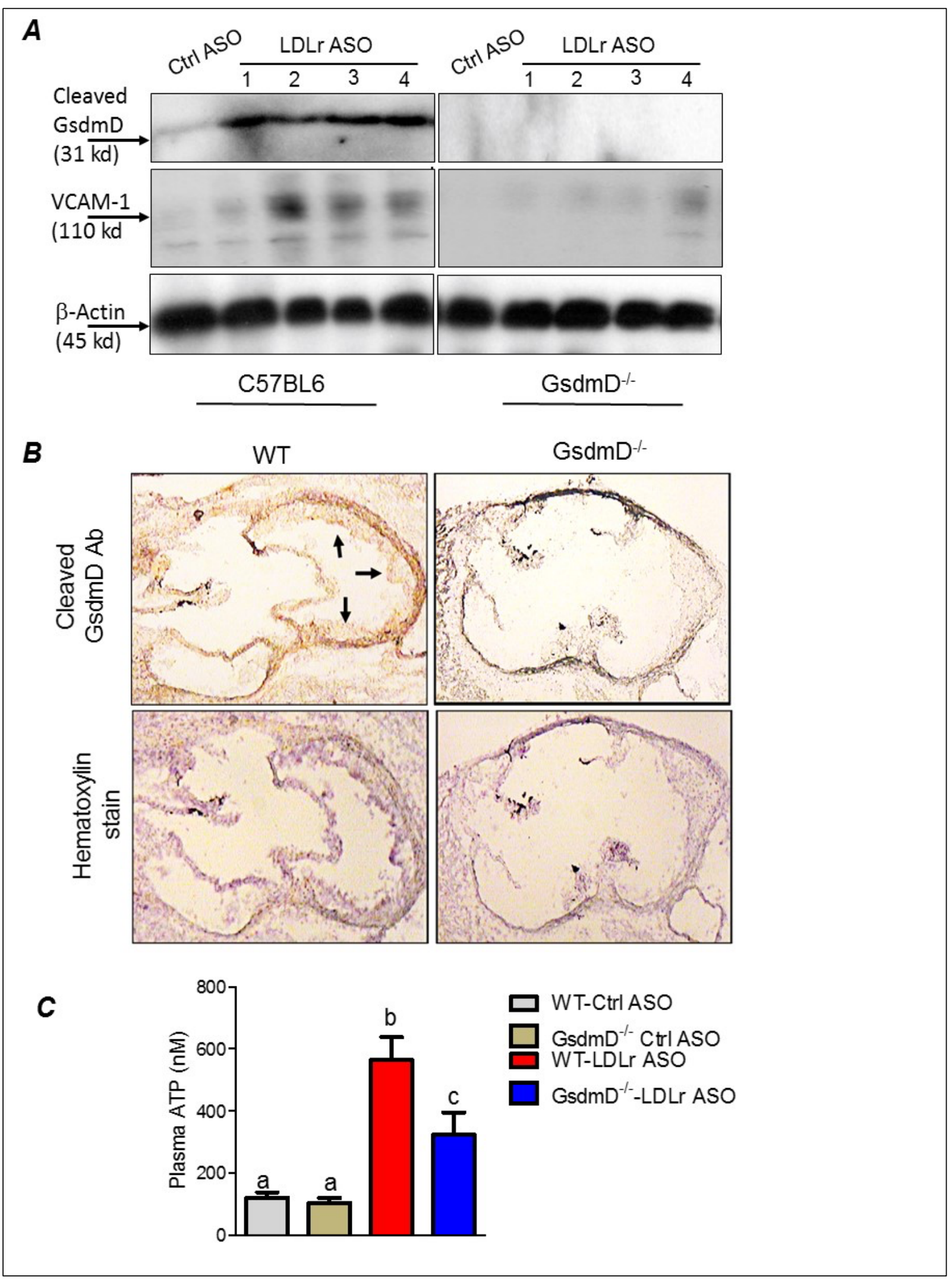

